\title{
The Vehicle Location Method Based on Artificial Neural Networks
}

\author{
Yingying Liu ${ }^{1, a}$, Zhi Kong ${ }^{2, b}$, Lifu Wang ${ }^{2, c}$, Yiyang Liu ${ }^{3, d}$ \\ ${ }^{1}$ School of Information Engineering, Shenyang University, Shenyang, 110044, China \\ ${ }^{2}$ School of Control of Engineering, Northeastern University At Qinhuangdao, Qinhuangdao, \\ Hebei, 066004, China \\ ${ }^{3}$ Shenyang Institute of Automation Chinese Academy of Sciences, Shenyang, 110016, China \\ a lyy3636@163.com, ${ }^{b}$ kongzhi2004916@163.com, c wlfkz@qq.com, dsialiuyiyang@sia.cn
}

Keywords: Location; GPS; Artificial neural network

Abstract. This paper introduces an GPS(Global Position Systems)location method based on articicial neural networks. Because the signal of GPS receiver is usually influenced surrounding environment, is difficult to obtain continuous and exact location data. A method based on Artificial Neural Network (ANN) is used to predict short time location information to guarantee real-time accurate navigation information. Simulation results have shown that the given method can obtain a certain precision and the approach is applicable.

\section{Introduction}

In recent years, vehicle location technology already has received the results in many ways. One of the most popular is the use of GPS for vehicle location, which provides global coverage, all day and free standard timing, navigation and positioning services under more favorable circumstances and gets high accuracy vehicle position and speed information.

However, this technique also has some defects [1-3]. The most important is the GPS signal will be influenced by high-rise buildings, tunnels, overpasses, trees and other surface features such as reflection and shadowing. GPS receiver in-car receives satellite signals with serious the multipath effect, therefore, only using GPS to achieve accurate continuous positioning is not possible. Based on consideration of these issues, it is hoping to obtain continuous position while using some other methods to make up for its shortcomings in order to ensure accurate positioning of the vehicle. Navigation system and map matching algorithms are employed by people often to complement with GPS positioning, to ensure continuous positioning. However, some of these methods need installing the more expensive instruments on the vehicle, moreover, map data processing often rely on real-time and accuracy [4-6]. Different form the papers mentioned above, this article use neural network method to predict results of GPS location within a certain distance to improve the positioning accuracy of GPS.

\section{Artificial neural network theory}

Artificial neural network is not necessary to solve the problem by modeling, but it is able to rebuild any nonlinear continuous function and can approach any of the functions with arbitrary precision. Using a neural network approach avoids the tedious routine modeling and neural network model has good adaptive and self-learning ability, strong anti-interference ability, and it makes neural networks obtain popular attention in predicting field.

Because of trees planted, tunnels and high-rise buildings and other factors, GPS signal of the vehicle is weak, GPS positioning system is not working properly. In this time, it can use of the results of the neural network of autonomous positioning to maintain normal navigation. In addition, when the system due to the small number of visible GPS satellite, positioning accuracy is low, but also the use of neural networks to predict results within a certain distance of a high accuracy to improve positioning accuracy of GPS. Therefore, the combination of GPS and artificial neural network can solve the low positioning accuracy problems of vehicle in a short time. This paper gives the framework of BP neural network 
model suitable for GPS positioning, uses conjugate gradient algorithm to improve the network's training speed and precision, and the algorithm is faster computing, is in favor of navigation and positioning of real-time data processing. Therefore, this GPS positioning algorithm based on neural network to obtain location information can improve timeliness and accuracy of location.

\section{Model and structure of neural network}

During the artificial network designing, we should determine the number of layers of the network, the number of neurons in each layer, the input and output points and activation function, and the initial value of the learning rate, etc. In this paper, we use BP neural network. Increase the number of layers is mainly to further reduce errors, improve accuracy, but also make the network complexity, increase the training time network weights and thresholds. In fact, to improve the accuracy can be achieved by increasing the number of neurons in the hidden layer to get their training effect is easier to observe and adjust than increasing the number of layers. It is generally considered to have just one hidden layer, this paper is the use of a hidden layer.

One part of the input is the receiver position coordinates of the last time, the other two parts of the input are the pseudo-range and pseudo-range correction value of satellite. The number of output nodes depends on the position coordinate components of the moment. We can apply of four satellites to obtain three-dimensional coordinates of the target. In this paper, we use 11 neurons in the input and 3 neurons in the output layer.

The number of neurons in the hidden layer can be obtained by the following formula:

$$
N_{2} \geq \log { }_{2}^{T}
$$

Where $N_{2}$ is the number of neurons in hidden layer; $T$ is the dimension of training samples.

The Network structure is given by Fig.1, including correction values of pseudo range at $t$ time (4 Variables), the pseudo range values at $t$ time (4 Variables), and the position coordinate components of receiver ( 3 Variables) input layer, 12 neurons in a a hidden layer and 3 neurons in the linear output layer standing for position coordinate components at $t$ time.

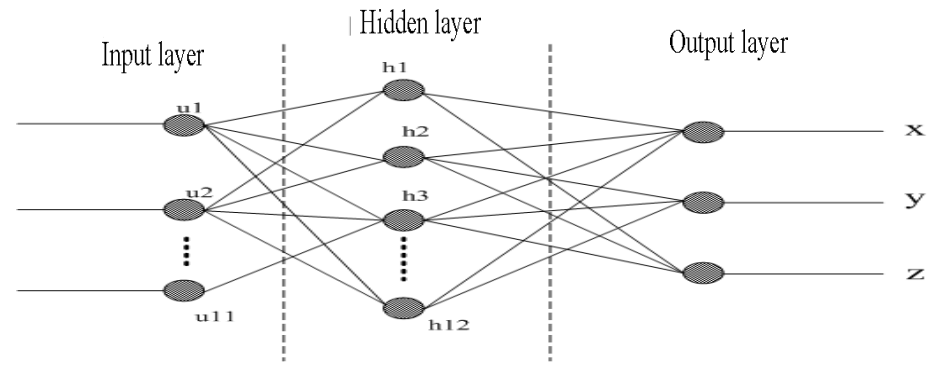

Fig 1. model configuration of BP networks

$\mathrm{BP}$ algorithm has two major problems in the actual use, that is slow convergence speed and local minima of the objective function. The approach of improvement usually has two kinds: using a heuristic learning method or using a more effective method of optimization. In this paper, we us the momentum method and the learning rate adaptive adjustment strategy provided by MATLAB toolbox to improve the learning speed and increase the reliability of the algorithm.

We use improved algorithm

$$
w(k+1)=w(k)+\alpha[(1-\eta) D(k)+\eta D(k-1)]
$$

where $w(k)$ is a single weight, also can be expressed as the negative gradient weight vector, $D(k)=\frac{-\partial E}{\partial w(k)}$ is the negative gradient of $\mathrm{K}$ moment, $D(k-1)$ is the negative gradient of $\mathrm{k}-1$ moment, $\alpha$ is the learning rate, $\alpha>0, \eta$ is momentum factor $0 \leq \eta \leq 1$, The added momentum can reduce the vibration trend of the learning process and improve the convergence of algorithm.

The improved algorithm uses adaptive learning rate:

$$
w(k+1)=w(k)+\alpha(k) D(k)
$$




$$
\begin{aligned}
& \alpha(k)=2 \lambda \alpha(k-1) \\
& \lambda=\operatorname{sign}[D(k) D(k-1)]
\end{aligned}
$$

\section{Simulation of GPS positioning model based on BP neural network}

BP artificial neural network nonlinear function approximation function is actually a generalized internal function interpolation. Thus, for a given navigation area, we should select an appropriate number of observation points in and around the central region, and more than one hour of continuous acquisition of sample data to train the network. This make thes network get more knowledge of the region, to improve the navigation and positioning accuracy. We use the coordinate data conversion software pieces will convert downloaded onto a Cartesian coordinate system.

We use the coordinate data conversion software pieces to convert downloaded navigation message into the data of cartesian coordinate. After obtaining the sample data vector, wherein the order of magnitude due to the difference of each index different from each other, each vector in the original sample is large, in order to facilitate the calculation part and prevents neuronal reached saturation, in the study of the sample input and output normalized treatment. The data processing for the data $[0,1]$. There are many forms of normalization method used here the following formula:

$$
x^{*}=\frac{x-x_{\min }}{x_{\max }-x_{\min }}
$$

Here we have chosen the 20 sets of data, of which 18 samples are the training group, the two groups are a test sample.

When using a single hidden layer BP network location prediction, we use 11 neurons 11-dimensional the input layer, 12 neurons in the middle layer, and three neurons in the output layer. BP network in accordance with the general design principles, the middle layer neuron transfer function can be set to the S-tangent function, the output has been normalized to the interval [0,1], and therefore, the output neuron transfer function can be set up as S-type logarithmic function.

When using a single hidden layer of BP network location prediction, since the input sample is 11-dimensional input vector, the input layer, a total of 11 neurons in the middle layer takes 12 neurons, the network has three output data, the output layer for three neurons, and therefore, should the network structure $11 * 12 * 3$. BP network in accordance with the general design principles, the middle layer neuron transfer function can be set to the $S$-tangent function, the output has been normalized to the interval [0,1], and therefore, the output neuron transfer function can be set up as S-type logarithmic function. The number of neurons in the middle layer is difficult to determine, which in turn significantly affect network performance prediction, first take the 12, and then observe the network performance, after going 10 and 15 respectively, and in this time compare the predicted performance, examine the effect of the number of neurons in the middle layer of the network performance. When the network's most-hour forecast error, the number of neurons in the middle layer of the network is the best value, where 12 is the best to take. BP neural network training results by using MATLAB have been shown in Figure 2.

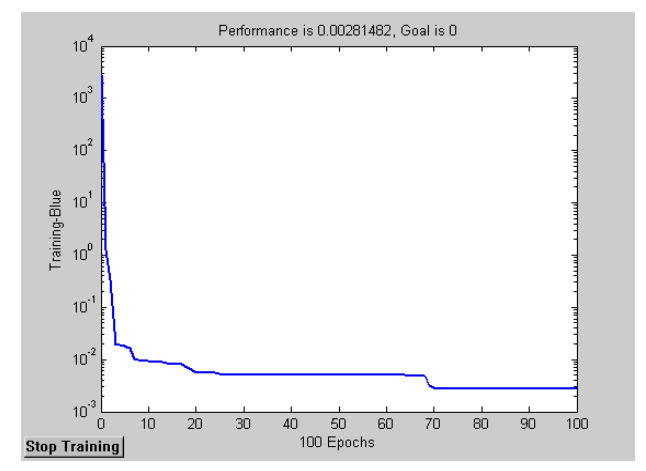

Fig 2. training process of BP networks 
The variables after 100 training target error network meet the requirements. After network training must also take advantage of the other two groups to test the positioning data, 19 sets of data and 20 sets of data and error test results are shown in Table 1 and Table 2 below:

Table 1. the result and error of the nineteenth group data

\begin{tabular}{|c|c|c|c|}
\hline & $X(\mathrm{~m})$ & $\mathrm{Y}(\mathrm{m})$ & $\mathrm{Z}(\mathrm{m})$ \\
\hline Actual value & 63.426915 & 10.41007 & 67.40 \\
\hline Predicted value & 63.375678 & 10.43967 & 67.53 \\
\hline Error & -0.051237 & 0.0296 & 0.13 \\
\hline \multicolumn{4}{|c|}{ Table 2. the result and error of the twentieth group data } \\
\hline & $\mathrm{X}(\mathrm{m})$ & $\mathrm{Y}(\mathrm{m})$ & $\mathrm{Z}(\mathrm{m})$ \\
\hline Actual value & 63.4269433 & 10.4100650 & 67.40 \\
\hline Predicted value & 63.4540413 & 10.4098563 & 67.43 \\
\hline Error & 0.0280980 & -0.0002087 & 0.03 \\
\hline
\end{tabular}

From the simulation, the GPS location method based on artificial neural network can effectively reduce the errors of location.

\section{Conclusion}

This paper provides a GPS navigation and position method based on the neural network. The method is easy to implement and can be processed in parallel, and obtains good performance and high positioning accuracy. The simulation results have shown that the GPS location method based on artificial neural network is effective and feasible.

\section{Acknowledgments}

This work is partially supported by the National Nature Science Foundation of China (Grant No.61104106, Grant No.61473195, Grant No. 61402088, Grant No. 61203329), Fundamental Research Funds for the Central Universities(Grant No. N130423013), Higher Educational Science and Technology Program of Hebei Province (Grant No. QN20132011), Science Research Foundation of Northeastern University at Qinhuangdao (Grant No.XNK201502), and Program Funded by Liaoning Province Education Administration (Grant No. L2014480, Grant No. L2015360).

\section{References}

[1] M Postorino, V Barrile, F Cotroneo. Surface movement ground control by means of a GPS-GIS system. Air Transport Management, 2006(12): 375-381.

[2] T H Wittea, A M Wilsona. Accuracy of non-differential GPS for the determination of speed over ground. Biomechanics, 2004(37): 1891-1898.

[3] Jitao Teng, Yuezu Fan. Analysis Method of Improving the Precision of Vehicle Location and Navigation in ITS. Proceedings of the 4th World Congress on Intelligent Control and Automation, 2002, 10(14): 1051-1054.

[4] Y. Tsai, F.Chang, W.Yang. GPS fault detection and exclusion using moving average filters. IEEE Proc.-Radar Sonar Navig. 2004, (4 ) : 789 795.

[5] Seshen Gao. Multi-sensor optimal data fusion for INS/GPS/SAR integrated navigation system. Aerospace Science and Technology, 2009, 13(4): 232-237.

[6] Haidong Hu , Xianlin Huang. SINS/CNS/GPS integrated navigation algorithm based on UKF, 
Journal of Systems Engineering and Electronics, 2010, 21(3):123-130. 